\title{
Perceived nervousness and moodiness associated with increased CVD but not cancer morbidity in pre- and postmenopausal women. Observations from the Population Study of Women in Gothenburg, Sweden
}

\section{Dominique Hange \\ Lauren Lissner \\ Calle Bengtsson \\ Valter Sundh \\ Cecilia Björkelund}

Sahlgrenska School of Public Health and Community Medicine, University of Gothenburg, Sweden
Correspondence: Dominique Hange Sahlgrenska School of Public Health and Community Medicine/Primary Health Care, PO Box 454 SE-405 30 Göteborg, Sweden

Tel +46317736843

Fax +4631778 I7 04

Email dominique.hange@vgregion.se

\begin{abstract}
A 32-year prospective observational study was initiated in 1968, including 1462 women aged $60,54,50,46$, and 38 , representative of the female population in these age groups. Measures included self-reported nervous symptoms and moodiness at baseline, menopausal status, risk factors as smoking, s-cholesterol, s-triglycerides, body mass index (BMI), waist-hip ratio (WHR), blood pressure, and socioeconomic status (SES). The prevalence of nervousness and moodiness was investigated as well as if these reported symptoms could predict mortality and morbidity within 32 years in pre-and postmenopausal women. Women who reported at least two of the symptoms suffered from an increased risk of 32-year mortality, independent of all background variables described above (hazard ratio $[\mathrm{HR}]=1.28$, confidence interval $[\mathrm{CI}]$ 1.03-1.58). Women with only one of the nervous symptoms already had an increased risk of suffering from cardiovascular disease (CVD) also after multivariable adjustment (HR = 1.29, CI 1.09-1.52) a risk that also remained significant when analyzing CVD risk in the group of premenopausal women $(\mathrm{HR}=1.28$, CI 1.02-1.62). There was no significantly increased risk among pre-or postmenopausal women with perceived nervousness/moodiness of developing cancer during the 32-year follow-up. There seems to be an association between nervousness/ moodiness and premature mortality and morbidity in CVD, especially when present already in the premenopausal state in women.
\end{abstract}

Keywords: Women, population study, nervousness, mortality, cardiovascular disease

\section{Introduction}

As early as in 1950, Terman and his colleagues reported that degree of psychological maladjustment, cumulatively rated, was related to higher risk of all-cause mortality over a four-decade follow-up period. ${ }^{1}$ The differences among causes of death were nonsignificant, but there was some indication that mental health problems were more strongly related to deaths from injury and cardiovascular disease (CVD). The overall relationship was significant for men but weaker for women. ${ }^{1}$

A recently published Swedish study, based on data from national surveys and registers, showed that perceived nervousness, uneasiness, and anxiety were strongly related to subsequent risks of suicide attempt and psychiatric disease as well as all-cause mortality, hospital care, and ischemic heart disease in both men and women. ${ }^{2}$

Several earlier studies in primary care have shown that depressed patients have impaired health-related quality of life (HRQoL) and that these patients score lower in this respect than patients with common chronic physical disorders. ${ }^{3}$ In some instances, specific psychoaffective patterns could be associated with particular 
physical diseases. For example depression has been linked to increased morbidity and mortality due to cardiac events ${ }^{4,5}$ and myocardial infarction ${ }^{6,7}$ whereas mixed anxiety-depression did not relate to excess mortality. ${ }^{8}$ However, there is an association between extreme anxiety and sudden cardiac death, where panic-like anxiety is an independent risk factor, ${ }^{9}$ as well as with increased mortality. ${ }^{7}$

Some studies have reported that depression increased the risk of death in elderly men but not in women, $8,10,11$ pointing to a gender difference in this association. It has long been recognized that women are at a higher risk than men to develop depression and that such risk is particularly associated with reproductive cycle events. ${ }^{12}$

There are not many studies concerning associations between nervousness/moodiness and cancer. Most studies report from patients who already have a cancer diagnosis and then relate the cancer diagnose to nervousness or mental stress. A Swedish study hypothesized that stressful psychosocial working conditions would be involved in the etiology of esophageal and gastric cardia cancers in men, but was unable to prove the hypothesis. ${ }^{13}$

The purpose of this study was to investigate if perceived nervousness and moodiness can predict mortality and morbidity within 32 years in women, and if so, independently of other well known risk factors as age, smoking, hypertension, s-cholesterol, s-triglycerides, body mass index (BMI), waist-hip ratio (WHR), socioeconomic status (SES), and education ${ }^{14,15}$ and also to relate possible associations to menopausal status. We used data from the Prospective Population Study of Women in Gothenburg.

\section{Participants and methods}

\section{Study population}

In 1968-1969, 1462 women aged 60, 54, 50, 46, and 38 in Gothenburg, Sweden, participated in the Prospective Population Study of Women in Gothenburg. The sampling was based on date of birth which together with the high participation rate $(90.1 \%)$, ensured that the participants were a representative cross-section of women from the community in the age groups studied. ${ }^{16}$ All women examined in 1968-1969 were offered examinations in 1974-1975, in 1980-1981, and in 1992-1993. In 2000-2001, the fifth examination, a 32-year follow-up study, with a participation rate of $70 \%$ among those still alive, took place. Details of the sampling procedure and participation rates at all examinations were presented elsewhere. ${ }^{16-20}$ We studied mortality and severe morbidity over 32 years and we used data from the National Cause of Death Register and the Swedish Hospital Patient
Register. The Swedish Hospital Patient Register contains data about all discharges from Swedish hospitals (overnight patients 1980-2001).

\section{Definition of nervousness and moodiness}

The presence of nervousness and moodiness was based on a self-administered questionnaire where nervousness was defined as answers yes to the question a) "Have you had any of the following illnesses/symptoms: psychological/nervous symptoms?" The women who reported having psychological/ nervous symptoms were defined as having nervousness.

The prevalence of moodiness was based on answers yes to one or both of the questions b1) "Do you usually feel downhearted and/or discouraged; at the moment/in the mornings/during the days/in the evenings?" or b2) "Regarding the problems of everyday life: Do you usually feel anxious and worried about them in advance?" All women reporting nervous symptoms and/or moodiness according to the above indicated questions were defined as belonging to the group with nervousness/moodiness (NM). Two different variables were used: i) NM1, corresponding to answering yes to at least one of the questions about the symptoms nervousness/ moodiness (a or b) (eg, "Yes, I feel downhearted in the mornings), and ii) NM2, corresponding to answering yes to at least two symptoms nervousness/moodiness (a and b) (eg, "Yes, I feel downhearted in the mornings" as well as "Yes, I have psychological/nervous symptoms").

\section{Definition of CVD and hypertension}

A woman was defined as having CVD if the diagnosis had been made by a doctor and was classified according to the International Classification of Diseases, Ninth and Tenth Revision (ICD 9 and 10), diagnosis codes ICD 391-448B or I01.0-I79.2, respectively (myocardial infarction, heart failure, other cardiovascular diseases, stroke). Risk time for CVD was calculated from the day the woman was admitted to hospital with this diagnosis (reliable data from Swedish Hospital Register was attained from 1980-2001). Women who reported having CVD (myocardial infarction or stroke) at start of the examinations in 1968-1969 were excluded. Hypertension/high blood pressure was defined as using antihypertensive medication and/or a systolic pressure $\geq 160 \mathrm{mmHg}$ and/or diastolic blood pressure $\geq 95 \mathrm{mmHg}$ at the time of the examination.

\section{Verification of cancer diagnosis}

We used data from the Swedish Cancer Registry and from regional cancer registries. Each participant was also asked if 
she had any history of neoplastic disease and, if so, she was asked to provide details on type, localization and treatment. Information from the total 32-year period 1968-1969 to 2000-2001 was obtained. Risk time for cancer was calculated to the month the woman got the diagnosis. A comparison between the 1988 Cancer Registry and the observations made in the Prospective Population Study of Women has shown that all but one out of 103 cases $(99 \%)$ were registered in the Cancer Registry and all but one (99\%) in the Prospective Population Study of Women. ${ }^{18}$ Women who reported having cancer at start of the examinations in 1968-1969 were excluded.

\section{Definition of menopausal status}

Menstrual status was obtained from a questionnaire and checked by interview by a physician. Information obtained in 1968-1969 was also verified retrospectively in 1974-1975. Menopause was defined as more than 12 months without menstruation. $^{21}$

\section{Measured CVD risk factors at baseline}

Physical examinations were conducted according to the same protocol at all examinations with respect to body weight, body height, BMI, waist circumference, hip circumference, WHR, systolic and diastolic blood pressure measurements (sitting position after five minutes' rest). ${ }^{16}$ Blood samples were drawn after an overnight fast. Serum levels of total cholesterol, s-triglycerides, and b-glucose were analyzed according to standard methods of the laboratory of the Sahlgrenska University Hospital, Gothenburg. These variables were entered as continuous covariates in the multivariate models.

\section{Social and lifestyle-related variables at baseline}

Women were classified as smokers (women who smoked or had stopped smoking during the previous year), ex-smokers (women who had stopped smoking more than a year before examination) and nonsmokers (women who never smoked). Women reported their own occupation and income and, if they were married, their husbands' occupation and income. This information was transformed according to Carlsson's standard occupations grouping system. ${ }^{22}$ We combined group one (large-scale employers and officials of high or intermediate rank) and groups two and three (small-scale employers, lower-rank officials, and supervisors) into "high and medium social class group." Groups four and five (skilled and unskilled workers) were classified as a "lower social class group." Women with only "basic" education (ca. six years, depending on cohort) were classified as less educated. Women reported about number of children. ${ }^{23}$ These variables were entered as categorical variables in the multivariate models.

\section{Statistical methods}

Fisher's exact test was used to test the hypothesis of no differences in prevalence of symptoms between the two groups (women with/without nervousness and /or moodiness in 1968-1969). Associations between mortality and morbidity and NM1 and NM2, respectively, were tested in Cox regression proportional hazards models with nervousness/ moodiness, smoking, s-cholesterol, s-triglycerides, BMI, WHR, hypertension, social group, education and number of children as independent variables. Since BMI as a risk factor is supposed to be J-shaped and presumed to have an irregular effect of the outcome variables, we included both a linear and quadratic effect of BMI in the regression models. The proportional hazards assumptions was tested and verified.

\section{Ethical approval and participants consent}

The Ethics Committee of University of Gothenburg approved the study. All subjects in the 2000-2001 examinations gave informed consent, in accordance with the provisions of the Helsinki Declaration.

\section{Results \\ Basal data on nervous symptoms and their association with mortality during a 32-year follow-up period}

Table 1 describes prevalence of nervousness and moodiness among the women in 1968-69, divided into different birth cohorts, as well as 32-year mortality. Around 60\% (862 participants) of the women reported not suffering from nervousness/moodiness with no significant differences between the different age cohorts $(\mathrm{p}=0.9)$ (data not shown). Within the 32 years, 524 women died. Table 1 also describes CVD and cancer morbidity in the different age groups 1968-2001.

Women who reported at least one of the symptoms nervousness and/or moodiness suffered from an increased mortality (39\% vs 33\%) during the 32-year follow-up period (Table 1). The association between two of the symptoms, nervousness and/or moodiness and mortality, was tested in a Cox proportional hazards model, showing a significantly increased risk when controlled for age, as well as after adjustment for all other baseline data (smoking, hypertension, 


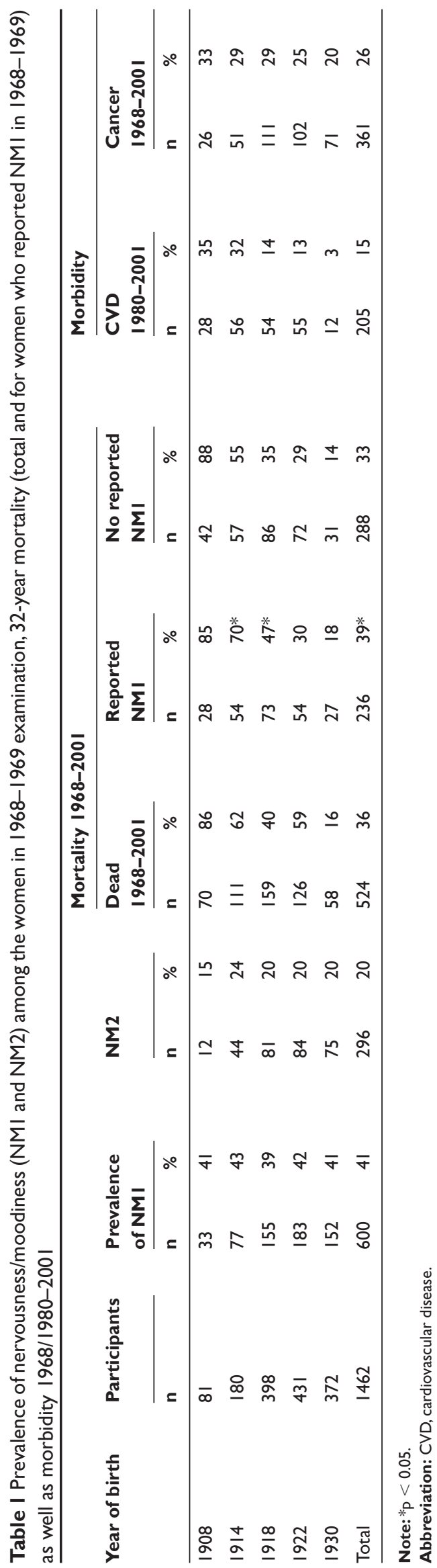

s-cholesterol, s-triglycerides, BMI, WHR, SES, education, number of children) (Table 2).

\section{Basal data on nervous symptoms and their association to incidence of CVD and cancer during a 32-year follow-up period}

\section{Cardiovascular disease}

There were totally 205 women who suffered from CVD, which had rendered stay overnight at hospital during 1980 until 2001 (Table 1). Women who had reported one of the symptoms of nervousness/moodiness in 1968-1969 had a significantly higher risk of CVD within 32 years, even after controlling for other risk factors (Table 3 ). Women who had reported at least two symptoms of nervousness/moodiness in 1968-1969 also had a significantly higher risk of CVD within 32 years, both when controlling for age, and with multivariable control (Table 3).

When studying the increased risk with regard to menopausal status in 1968-1969 women in the premenopausal group, with reported nervousness and/or moodiness, showed significantly increased risk of CVD also after multivariable adjustment (Table 3). Concerning the postmenopausal group, women who reported at least one of the symptoms nervousness/moodiness showed significantly increased risk of CVD when controlling for age, but not multivariable controlled (Table 3).

Most important risk factors in the models were WHR, smoking and s-triglycerides; for the premenopausal group: WHR and smoking, and for the postmenopausal group: WHR, smoking and triglycerides. Introducing one risk factor at a time revealed no factor more prominent than the other. Belonging to a high social group was a protective factor.

\section{Cancer}

There were 361 women who suffered from cancer within 32 years (Table 1). Women with reported nervousness/moodiness did not suffer from cancer in a higher degree than women without these symptoms in 1968-1969 (Tables 2 and 3).

\section{Discussion}

In the present study, women with perceived nervousness/ moodiness in 1968-1969 showed a significantly increased risk of mortality over 32 years. A statistically increased risk of suffering from CVD remained in premenopausal women, with perceived nervousness/moodiness, even 
Table 2 Association between nervousness/moodiness (NMI and NM2) and risk of death in a 32-year perspective; all women divided into two groups, controlled for age and all risk factors, respectively. Total mortality, CVD mortality, and cancer mortality are presented

\begin{tabular}{lllll}
\hline & $\mathbf{n}$ & HR & Cl & $\mathbf{p}$ \\
\hline Total mortality & & & & \\
NMI + age & 1347 & 1.18 & $0.98-1.42$ & 0.07 \\
Multivariable & 1347 & 1.12 & $0.94-1.35$ & 0.21 \\
NM2 + age & 1347 & 1.39 & $1.13-1.72$ & 0.002 \\
Multivariable & 1347 & 1.28 & $1.03-1.58$ & 0.02 \\
CVD mortality & & & & \\
NMI + age & 1347 & 1.38 & $1.04-1.83$ & 0.03 \\
Multivariable & 1347 & 1.33 & $1.00-1.77$ & 0.05 \\
NM2 + age & 1347 & 1.58 & $1.14-2.18$ & 0.005 \\
Multivariable & 1347 & 1.47 & $1.06-2.04$ & 0.02 \\
Cancer mortality & & & & \\
NMI + age & 1347 & 0.86 & $0.61-1.20$ & 0.37 \\
Multivariable & 1347 & 0.82 & $0.58-1.14$ & 0.24 \\
NM2 + age & 1347 & 0.90 & $0.59-1.38$ & 0.63 \\
Multivariable & 1347 & 0.83 & $0.54-1.27$ & 0.39 \\
\hline
\end{tabular}

Abbreviations: CVD, cardiovascular disease; $\mathrm{HR}$, hazards ratio; $\mathrm{Cl}$, confidence interval.

after controlling for well known risk factors, including socioeconomic status and lifestyle factors. Women who reported perception of least two symptoms of nervousness/moodiness showed increased risk of both total and CVD mortality, as well as earlier found association with increased CVD morbidity in the group of women premenopausal in 1968-1969.

A previous study of Swedish men and women showed an association between nervousness and anxiety and 10-year mortality. Specifically there were much stronger associations among men and with no significant association concerning ischemic heart disease in women and much weaker associations concerning female mortality and morbidity over all. ${ }^{2}$ In our longitudinal study, the women, representative of the population, were thoroughly examined at baseline, which made it possible to control for several known risk factors, both concerning socioeconomic status and education as well as lifestyle variables.

Perceived nervousness and moodiness seems to be associated with increased risk of suffering from CVD, especially in premenopausal women. This in a way contradicts many studies which have shown that estrogen deficiency after spontaneous or medically induced menopause is an important risk factor for CVD. ${ }^{24}$ On the
Table 3 Association between nervousness/moodiness (NMI and NM2) and CVD and cancer morbidity, respectively, in a 32-year perspective; all women presented as well as divided in groups for premenopausal and postmenopausal in 1968-1969. Calculations controlled for age as well as multivariable controlled presented

\begin{tabular}{|c|c|c|c|c|}
\hline CVD morbidity & $n$ & HR & $\mathrm{CI}$ & $\mathbf{p}$ \\
\hline \multicolumn{5}{|l|}{ All women } \\
\hline $\mathrm{NMI}+$ age & 1344 & 1.32 & I.12-I.56 & 0.001 \\
\hline Multivariable & 1344 & 1.29 & I.09-1.52 & 0.003 \\
\hline $\mathrm{NM} 2+$ age & 1344 & 1.32 & $1.08-1.60$ & 0.006 \\
\hline Multivariable & 1344 & 1.27 & I.04-I.54 & 0.02 \\
\hline \multicolumn{5}{|c|}{$\begin{array}{l}\text { Women premenopausal } \\
\text { in 1968-1969 }\end{array}$} \\
\hline $\mathrm{NMI+}$ age & 828 & 1.28 & $1.02-1.61$ & 0.03 \\
\hline Multivariable & 828 & 1.28 & $1.02-1.62$ & 0.03 \\
\hline $\mathrm{NM} 2$ + age & 828 & 1.46 & $1.10-1.93$ & 0.009 \\
\hline Multivariable & 828 & 1.44 & $1.08-1.91$ & 0.01 \\
\hline \multicolumn{5}{|c|}{$\begin{array}{l}\text { Women postmenopausal } \\
\text { in 1968-1969 }\end{array}$} \\
\hline $\mathrm{NMI}+$ age & 449 & 1.32 & I.03-1.70 & 0.03 \\
\hline Multivariable & 449 & 1.21 & $0.94-1.56$ & 0.13 \\
\hline NM2 + age & 449 & 1.10 & $0.83-1.47$ & 0.49 \\
\hline Multivariable & 449 & 1.02 & $0.76-1.36$ & 0.89 \\
\hline \multicolumn{5}{|l|}{ Cancer morbidity } \\
\hline $\mathrm{NMI}+$ age & 1345 & 1.02 & $0.82-1.28$ & 0.86 \\
\hline Multivariable & 1345 & 1.00 & $0.80-1.25$ & 0.98 \\
\hline NM2 + age & 1345 & 1.09 & $0.83-1.42$ & 0.55 \\
\hline Multivariable & 1345 & 1.05 & $0.80-1.38$ & 0.72 \\
\hline \multicolumn{5}{|c|}{$\begin{array}{l}\text { Women premenopausal } \\
\text { in 1968-1969 }\end{array}$} \\
\hline $\mathrm{NMI}+$ age & 828 & 1.18 & $0.87-1.58$ & 0.28 \\
\hline Multivariable & 828 & 1.14 & $0.84-1.53$ & 0.40 \\
\hline NM2 + age & 828 & 1.13 & $0.78-1.66$ & 0.50 \\
\hline Multivariable & 828 & 1.08 & $0.74-1.57$ & 0.69 \\
\hline \multicolumn{5}{|c|}{$\begin{array}{l}\text { Women postmenopausal } \\
\text { in } 1968-1969\end{array}$} \\
\hline $\mathrm{NMI}+$ age & 450 & 0.90 & $0.62-1.30$ & 0.57 \\
\hline Multivariable & 450 & 0.92 & $0.63-1.34$ & 0.65 \\
\hline NM2 + age & 450 & 1.02 & $0.66-1.58$ & 0.93 \\
\hline Multivariable & 450 & 1.03 & $0.67-1.60$ & 0.89 \\
\hline
\end{tabular}

Abbreviations: $\mathrm{HR}$, hazards ratio; $\mathrm{Cl}$, confidence interval.

other hand, an increased risk of stroke among younger women (30-60 years) has been observed in Sweden during the last decades..$^{25}$ This could indicate that younger, premenstrual women in our society have a higher susceptibility to eg, mental stress. The influence of ovarian hormones on the stress and immune responses during the reproductive stages in women's lives is still not fully understood. ${ }^{26}$ There seems to be no simple explanation. 
Estrogen has been used in the treatment of adult-onset depressive and anxiety symptoms in postmenopausal women, most often with little or no effect on symptoms. ${ }^{27}$ Hormone replacement therapy (HRT) use in 1968 in 50-year-old women was very low; $2 \%$ of 50 -year-old women and $5 \%$ of 54-year-old women used HRT during more than a month in the 1968-1969 year examination. ${ }^{21}$ This makes any influence of importance on presented results unlikely.

Most associations found between nervousness/moodiness and mortality as well as morbidity could be explained in several ways, eg, by association of the symptoms with other health related conditions. Smoking is more prevalent in persons with psychological/mental distress. ${ }^{28}$ Depressive symptoms and stressful life events have been found to be associated with the metabolic syndrome, eg abdominal adiposity and high s-triglycerides, in the Healthy Women's Study. ${ }^{29}$ The association between morbidity and mortality indicators and low socioeconomic status has been observed for many centuries and previously reported in this cohort. ${ }^{30}$ Certain studies have shown that lower social class is associated with a high frequency and severity of perceived distress. ${ }^{31,32}$ As always, cause or effect cannot be revealed by observational studies, not even population based prospective studies, and we can only speculate if the association between perceived nervousness/moodiness and CVD morbidity is a direct or indirect effect via increased risk contributable to eg, smoking, psychosocial stress, or poor lifestyle.

The advantages of this study is the longitudinal design and the high participation rate following the women in the original study in 1968-1969 until the follow-up study in 2000-2001, using exactly the same examination protocol in all follow-ups.

There are some limitations to this study. We used the women's own perception of nervousness/moodiness rather than a more sophisticated medical instrument or doctors' diagnoses. The psychological symptoms were subjectively and individually reported. On the other hand, a symptom is a symptom and not a diagnosis or an illness and it is only the person herself who can state if she perceives the symptom or not. The corresponding way of documenting nervousness was also used in the reported Swedish study. ${ }^{2}$ Prevalence of at least one symptom of nervousness or moodiness was higher in our study compared to that study, but corresponding figures were reached when using the variable at least two symptoms nervousness/moodiness.

In conclusion, this study shows that there seems to exist an association between nervousness/moodiness and morbidity, especially concerning CVD morbidity in premenopausal women, but the associations could to some extent be explained by background variables. This could be another example of the close association between socioeconomic and psychosocial factors, where cause or effect cannot be determined.

\section{Acknowledgments}

This study was supported by grants from Swedish Research Council and the Swedish Council for Life and Social Research (EpiLife, FAS WISH). The authors declare no conflicts of interest exist in this work.

\section{References}

1. Martin LR, Friedman HS, Tucker JS, et al. An archival prospective study of mental health and longevity. Health Psychol. 1995;14(5):381-387.

2. Ringback Weitoft G, Rosen M. Is perceived nervousness and anxiety a predictor of premature mortality and severe morbidity? A longitudinal follow up of the Swedish survey of living conditions. J Epidemiol Community Health. 2005;59(9):794-798.

3. Hays RD, Wells KB, Sherbourne CD, Rogers W, Spritzer K. Functioning and well-being outcomes of patients with depression compared with chronic general medical illnesses. Arch Gen Psychiatry. 1995;52(1):11-19.

4. Strik JJ, Honig A, Maes M. Depression and myocardial infarction: relationship between heart and mind. Prog Neuropsychopharmacol Biol Psychiatry. 2001;25(4):879-892.

5. Barth J, Schumacher M, Herrmann-Lingen C. Depression as a risk factor for mortality in patients with coronary heart disease: a meta-analysis. Psychosom Med. 2004;66(6):802-813.

6. Pratt LA, Ford DE, Crum RM, Armenian HK, Gallo JJ, Eaton WW. Depression, psychotropic medication, and risk of myocardial infarction. Prospective data from the Baltimore ECA follow-up. Circulation. 1996;94(12):3123-3129.

7. Stoner SM, PA. Sommi RW. Psychiatric Comorbidity and Medical Illness. Medical Update for Psychiatrists. 1998;3:64-70.

8. Holwerda TJ, Schoevers RA, Dekker J, Deeg DJ, Jonker C, Beekman AT. The relationship between generalized anxiety disorder, depression and mortality in old age. Int J Geriatr Psychiatry. 2007;22(3):241-249.

9. Fleet RP, Beitman BD. Cardiovascular death from panic disorder and panic-like anxiety: a critical review of the literature. J Psychosom Res. 1998;44(1):71-80.

10. Mallon L, Broman JE, Hetta J. Relationship between insomnia, depression, and mortality: a 12-year follow-up of older adults in the community. Int Psychogeriatr. 2000;12(3):295-306.

11. Schoevers RA, Geerlings MI, Beekman AT, et al. Association of depression and gender with mortality in old age. Results from the Amsterdam Study of the Elderly (AMSTEL). Br J Psychiatry. 2000;177:336-342.

12. Frey BN, Lord C, Soares CN. Depression during menopausal transition: a review of treatment strategies and pathophysiological correlates. Menopause Int. 2008;14(3):123-128.

13. Jansson C, Johansson AL, Jeding K, Dickman PW, Nyren O, Lagergren J. Psychosocial working conditions and the risk of esophageal and gastric cardia cancers. Eur J Epidemiol. 2004;19(7):631-641.

14. Porgeirsson G, Davidsson D, Sigvaldason H, Sigfusson N. Coronary risk factors among men and women in Iceland. Results from the Reykjavik Study 1967-1985. 1992. Laeknabladid. 2005;91(1):107-114.

15. Baldwin D. Depression and panic: Comorbidity. European Psychiatry. 1998;13:65-70.

16. Bengtsson $\mathrm{C}$, Blohmé G, Hallberg L, et al. The study of women in Gothenburg 1968-1969-a population study. General design, purpose and sampling results. Acta Med Scand. 1973;193(4):311-318. 
17. Bengtsson C, Hallberg L, Hällstrom T, et al. The population study of women in Goteborg 1974-1975-the second phase of a longitudinal study. General design, purpose and sampling results. Scand J Soc Med. 1978;6(2):49-54.

18. Bengtsson C, Gredmark T, Hallberg L, et al. The population study of women in Gothenburg 1980-81-the third phase of a longitudinal study. Comparison between participants and non-participants. Scand J Soc Med. 1989;17(2):141-145.

19. Bengtsson C, Ahlqwist M, Andersson K, Björkelund C, Lissner L, Söderström M. The Prospective Population Study of Women in Gothenburg, Sweden, 1968-69 to 1992-93. A 24-year follow-up study with special reference to participation, representativeness, and mortality. Scand J Prim Health Care. 1997;15(4):214-219.

20. Lissner L, Skoog I, Andersson K, et al. Participation bias in longitudinal studies: experience from the Population Study of Women in Gothenburg, Sweden. Scand J Prim Health Care. 2003;21(4):242-247.

21. Rödstrom K, Bengtsson C, Lissner L, Björkelund C. Pre-existing risk factor profiles in users and non-users of hormone replacement therapy: prospective cohort study in Gothenburg, Sweden. BMJ. 1999;319(7214):890-893.

22. Carlsson G. Social mobility and class structure. Lund, Sweden: CWK Gleerup; 1958.

23. Grundy E, Kravdal O. Reproductive history and mortality in late middle age among Norwegian men and women. Am J Epidemiol. 2008;167(3):271-279.

24. Gorodeski GI. Update on cardiovascular disease in post-menopausal women. Best Pract Res Clin Obstet Gynaecol. 2002;16(3):329-355.
25. Medin J, Nordlund A, Ekberg K. Increasing stroke incidence in Sweden between 1989 and 2000 among persons aged 30 to 65 years: evidence from the Swedish Hospital Discharge Register. Stroke. 2004;35(5):1047-1051.

26. Motzer SA, Hertig V. Stress, stress response, and health. Nurs Clin North Am. 2004;39(1):1-17.

27. Morrison MF, Kallan MJ, Ten Have T, Katz I, Tweedy K, Battistini M. Lack of efficacy of estradiol for depression in postmenopausal women: a randomized, controlled trial. Biol Psychiatry. 2004;55(4): 406-412.

28. Sacker A, Bartley M, Firth D, Fitzpatrick R. Dimensions of social inequality in the health of women in England: occupational, material and behavioural pathways. Soc Sci Med. 2001;52(5):763-781.

29. Hunt SM, McEwen J, McKenna SP. Social inequalities and perceived health. Eff Health Care. 1985;2(4):151-160.

30. Cabrera C, Hakeberg M, Ahlqwist M, et al. Can the relation between tooth loss and chronic disease be explained by socio-economic status? A 24-year follow-up from the population study of women in Gothenburg, Sweden. Eur J Epidemiol. 2005;20(3):229-236.

31. Strine TW, Balluz L, Chapman DP, Moriarty DG, Owens M, Mokdad AH Risk behaviors and healthcare coverage among adults by frequent mental distress status, 2001. Am J Prev Med. 2004;26(3):213-216.

32. Raikkonen K, Matthews KA, Kuller LH. Depressive symptoms and stressful life events predict metabolic syndrome among middle-aged women: a comparison of World Health Organization, Adult Treatment Panel III, and International Diabetes Foundation definitions. Diabetes Care. 2007;30(4):872-877. 
